\title{
Flavonol Galactoside Caffeiate Ester and Homoisoflavones from Caesalpinia millettii Hook. et ARN.
}

\author{
Ping CHEN and Jun-Shan YANG* \\ Institute of Medicinal Plant Development, Chinese Academy of Medical Sciences and Peking Union Medical College; \\ Beijing 100094, P. R. China. Received November 3, 2006; accepted December 14, 2006
}

\begin{abstract}
Chemical examination of the stems of Caesalpinia millettii Hook. et ARN led to the isolation of new flavonol glycoside caffeiate ester (1) and homoisoflavone (2), along with four known homoisoflavones: eucomin (3), bonducellin (4), 8-methoxybonducellin (5) and intricatinol (6). The structures of 1 and 2 were established to be tamarixetin 3-O-(6"-O-E-caffeoyl)- $\beta$-D-galactopyranoside (1) and (Z)-7-hydroxy-8-methoxy-3-(4-methoxybenzyl) chroman-4-one (2) on the basis of detailed analyses of physical, chemical, and spectral data. Compounds $3-6$ were isolated from this plant for the first time.
\end{abstract}

Key word Caesalpinia millettii; tamarixetin; homoisoflavone; 8-methoxyisobonducellin; flavonol glycoside caffeiate ester

Seventeen species of the Caesalpinia genus (Leguminosae) are discovered in southwestern China, ${ }^{1)}$ and they possess similar morphologic characteristics. However, the reports on the chemical structure of the Caesalpinia genus indicate they have different chemical components showing various bioactivities. Thus, further investigation of the chemical constituent of Caesalpinia genus is worthwhile.

Caesalpinia millettii HoOK. et ARN. is a thorny liana of the family Leguminosae, which is widely distributed in Yunnan Province, Guangxi Province, Guangdong Province of China. Up to now, there is no any report regarding the constituents of it. In this paper we describes the isolation and structural elucidation of the new flavonol glycoside caffeiate ester (1) and homoisoflavone (2), as well as four known homoisoflavones (3-6) from the acetone extract of the stems of Caesalpinia millettii HooK. et ARN. The structures of the known homoisoflavones $(3-6)$ were identified as $(E)-5,7-$ dihydroxy-3-(4-methoxybenzyl) chroman-4-one (3), (E)-7hydroxy-3-(4-methoxybenzyl) chroman-4-one (4), (E)-7-hydroxy-8-methoxy-3-(4-methoxybenzyl) chroman-4-one (5), (E)-7,8-dihydroxy-3-(4-methoxybenzyl) chroman-4-one (6),
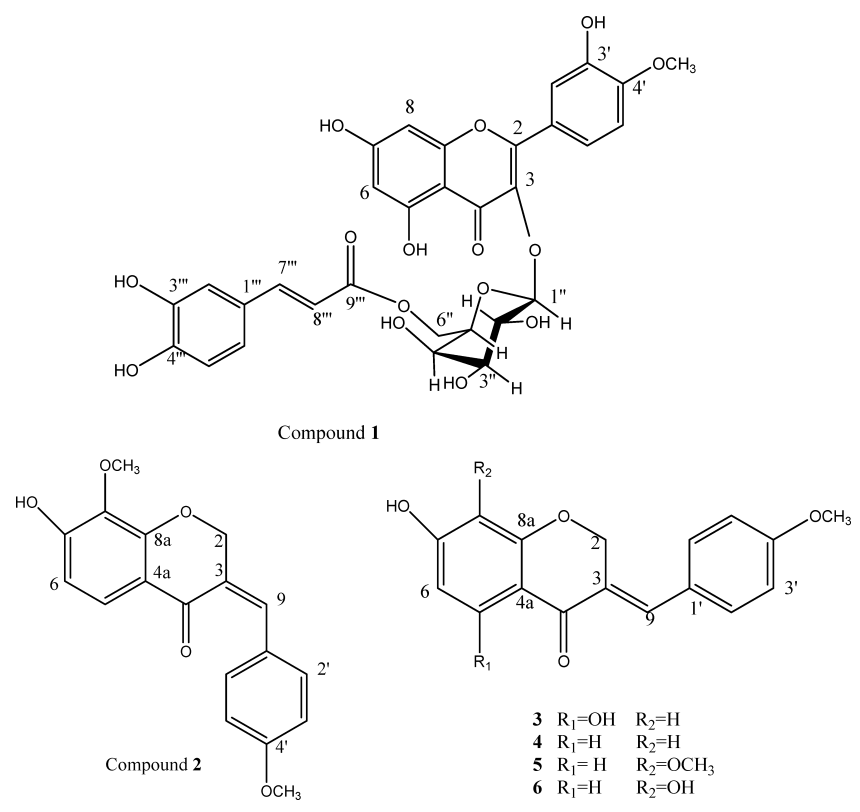

Fig. 1. Structures of Compounds $\mathbf{1}-\mathbf{6}$ respectively.

\section{Results and Discussion}

Compound $\mathbf{1}$ was obtained as yellow powder, gave a positive coloration in the $\mathrm{Mg}-\mathrm{HCl}$ test. The molecular formula was determined as $\mathrm{C}_{31} \mathrm{H}_{28} \mathrm{O}_{15}$, on the basis of the ion peak $[\mathrm{M}+\mathrm{Na}]^{+}$at $m / z$ 663.1311 (Calcd 663.1325) in HR-FABMS. The UV spectrum showed maximum absorption at 295 and $330 \mathrm{~nm}$, and the IR spectrum exhibited absorption bands ascribed to hydroxyl $\left(3250 \mathrm{~cm}^{-1}\right)$, ester carbonyl (1645 $\left.\mathrm{cm}^{-1}\right)$, and aromatic ring $\left(1604,1508 \mathrm{~cm}^{-1}\right)$. Thus, 1 was suggested to be flavonol glycoside from its characteristic color reaction and spectral properties. Analysis of the ${ }^{1} \mathrm{H}$ and ${ }^{13} \mathrm{C}-\mathrm{NMR}$ spectra of compound $\mathbf{1}$ (Table 1; all assignments based on HMQC, HMBC experiments) showed the presence of a tamarixetin core, a trans-caffeoyl moiety, and a galactopyranosyl unit. The ${ }^{1} \mathrm{H}-\mathrm{NMR}$ resonances of two meta coupled doublets at $\delta 6.10(1 \mathrm{H}, \mathrm{d}, J=2.0 \mathrm{~Hz}, \mathrm{H}-6)$ and 6.34 $(1 \mathrm{H}, \mathrm{d}, J=2.0 \mathrm{~Hz}, \mathrm{H}-8)$, correlated with the carbons at 99.35 and 94.13 ppm respectively in the HMQC spectrum, characterized the 6- and 8-protons of a flavonol 5, 7 dihydroxy Aring. ${ }^{9)}$ And $\delta 6.80\left(1 \mathrm{H}, \mathrm{d}, J=8.5 \mathrm{~Hz}, \mathrm{H}-5^{\prime}\right), 7.50(1 \mathrm{H}, \mathrm{d}, J=$ $\left.2.0 \mathrm{~Hz}, \mathrm{H}-2^{\prime}\right)$, and $7.65\left(1 \mathrm{H}, \mathrm{dd}, J=8.5,2.0 \mathrm{~Hz}, \mathrm{H}-6^{\prime}\right)$, correlated with the carbons at $115.88,116.57$ and $122.65 \mathrm{ppm}$ respectively in the HMQC spectrum, characterized the ABX system due to $3^{\prime}, 4^{\prime}$-disubstituted B-ring. A singlet signal of three protons at $\delta 3.80$ correlated with the carbons at $149.17 \mathrm{ppm}$ and $7.50\left(\mathrm{H}-2^{\prime}\right), 7.65\left(\mathrm{H}-6^{\prime}\right)$ correlated with the carbons at $149.17 \mathrm{ppm}$ in the $\mathrm{HMBC}$ (see Fig. 2) indicated the presence of the flavonol 3'-hydroxy-4'-methoxy substituted B-ring. Thus, the aglycone part of 1 was analyzed as tamarixetin. In the aromatic region of the spectrum, the remaining three protons occurred as an ABX spin system at $\delta$ $6.76\left(1 \mathrm{H}, \mathrm{d}, J=8.0 \mathrm{~Hz}, \mathrm{H}-5^{\prime \prime \prime}\right), 6.91\left(1 \mathrm{H}, \mathrm{d}, J=8.0 \mathrm{~Hz}, \mathrm{H}-6^{\prime \prime \prime}\right)$ and $7.19\left(1 \mathrm{H}\right.$, brs, $\left.\mathrm{H}-2^{\prime \prime \prime}\right)$, and the double bond protons $(\delta$ $\left.6.23,1 \mathrm{H}, J=16 \mathrm{~Hz}, \mathrm{H}-8^{\prime \prime \prime}\right),\left(\delta 7.34,1 \mathrm{H}, J=16 \mathrm{~Hz}, \mathrm{H}-7^{\prime \prime \prime}\right)$ for the trans-caffeoyl moiety. In the HMBC spectrum, the caffeoyl carbonyl group ( $\delta$ 166.93) correlated not only with the double bond protons $(\delta 6.23,7.34)$ but also with the 6 -methylene protons $(\delta 4.08)$, suggesting that the caffeoyl moiety is connected to the 6-methylene carbon of the galactose. Moreover, the cross peak observed between $\mathrm{C}-3$ ( $\delta$ 134.11) and the anomeric proton $(\delta 5.39, \mathrm{~d}, J=8.0 \mathrm{~Hz})$ showed that the 
Table $1 .{ }^{13} \mathrm{C}$ - and ${ }^{1} \mathrm{H}-\mathrm{NMR}\left(500 \mathrm{MHz}, 125 \mathrm{MHz}\right.$, DMSO- $\left.d_{6}\right)$ Data of Compound 1

\begin{tabular}{|c|c|c|c|c|}
\hline \multirow{2}{*}{ No. } & \multicolumn{2}{|c|}{ 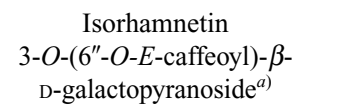 } & \multicolumn{2}{|r|}{1} \\
\hline & $\delta_{\mathrm{C}}$ & $\delta_{\mathrm{H}}$ & $\delta_{\mathrm{C}}$ & $\delta_{\mathrm{H}}$ \\
\hline 2 & 157.3 & - & 156.93 & - \\
\hline 3 & 133.7 & - & 134.11 & - \\
\hline 4 & 177.7 & - & 178.12 & - \\
\hline 5 & 161.4 & - & 161.84 & - \\
\hline 6 & 99.2 & 6.12 br s & 99.35 & $6.10(\mathrm{~d}, 2.0 \mathrm{~Hz})$ \\
\hline 7 & 166.3 & - & 164.78 & - \\
\hline 8 & 93.9 & 6.27 br s & 94.13 & $6.34(\mathrm{~d}, 2.0 \mathrm{~Hz})$ \\
\hline 9 & 157.0 & - & 156.93 & - \\
\hline 10 & 103.7 & - & 104.50 & - \\
\hline $1^{\prime}$ & 121.5 & - & 121.74 & - \\
\hline $2^{\prime}$ & 113.0 & $7.82(\mathrm{~d}, 1.5 \mathrm{~Hz})$ & 116.57 & $7.50(\mathrm{~d}, 2.0 \mathrm{~Hz})$ \\
\hline $3^{\prime}$ & 146.9 & - & 145.55 & - \\
\hline $4^{\prime}$ & 149.4 & - & 149.17 & - \\
\hline $5^{\prime}$ & 112.8 & $6.80(\mathrm{~m})$ & 115.88 & $6.80(\mathrm{~d}, 8.5 \mathrm{~Hz})$ \\
\hline $6^{\prime}$ & 122.5 & $7.54(\mathrm{dd}, 8.5,1.5 \mathrm{~Hz})$ & 122.65 & $7.65(\mathrm{dd}, 8.5,2.0 \mathrm{~Hz})$ \\
\hline $1^{\prime \prime}$ & 102.7 & $5.24(\mathrm{~d}, 7 \mathrm{~Hz})$ & 102.29 & $5.39(\mathrm{~d}, 8.0 \mathrm{~Hz})$ \\
\hline $2^{\prime \prime}$ & 74.4 & $3.47(\mathrm{~m})$ & 71.71 & $3.57(1 \mathrm{H}, \mathrm{m})$ \\
\hline $3^{\prime \prime}$ & 74.4 & $3.50(\mathrm{~m})$ & 73.55 & $3.43(1 \mathrm{H}, \mathrm{m})$ \\
\hline $4^{\prime \prime}$ & 70.4 & $3.33(\mathrm{~m})$ & 68.99 & $3.66(1 \mathrm{H}, \mathrm{m})$ \\
\hline $5^{\prime \prime}$ & 76.6 & $3.47(\mathrm{~m})$ & 73.67 & $3.68(1 \mathrm{H}, \mathrm{m})$ \\
\hline $6^{\prime \prime}$ & 62.8 & $\begin{array}{l}4.27(\mathrm{dd}, 2,11.8 \mathrm{~Hz}) \\
4.22(\mathrm{dd}, 7,11.8 \mathrm{~Hz})\end{array}$ & 63.82 & $4.08(2 \mathrm{H}, \mathrm{m})$ \\
\hline $1^{\prime \prime \prime}$ & 126.2 & - & 126.14 & - \\
\hline $2^{\prime \prime \prime}$ & 113.0 & 6.94 (brs) & 111.70 & $7.19(\mathrm{~d}, 2.0 \mathrm{~Hz})$ \\
\hline $3^{\prime \prime \prime}$ & 145.3 & - & 148.55 & - \\
\hline $4^{\prime \prime \prime}$ & 148.2 & - & 150.00 & - \\
\hline $5^{\prime \prime \prime}$ & 115.1 & $6.78(\mathrm{~m})$ & 116.13 & $6.76(\mathrm{~d}, 8.0 \mathrm{~Hz})$ \\
\hline $6^{\prime \prime \prime}$ & 121.8 & $6.78(\mathrm{~m})$ & 123.73 & $6.91(\mathrm{dd}, 8.0,2.0 \mathrm{~Hz})$ \\
\hline $7^{\prime \prime \prime}$ & 145.7 & $7.30(\mathrm{~d}, 16 \mathrm{~Hz})$ & 145.75 & $7.34(\mathrm{~d}, 16 \mathrm{~Hz})$ \\
\hline $8^{\prime \prime \prime}$ & 113.7 & $5.98(\mathrm{~d}, 16 \mathrm{~Hz})$ & 114.60 & $6.23(\mathrm{~d}, 16 \mathrm{~Hz})$ \\
\hline $9^{\prime \prime \prime}$ & 167.5 & - & 166.93 & - \\
\hline $\mathrm{OMe}$ & 55.2 & $3.89(\mathrm{~s})$ & 56.33 & $3.79(\mathrm{~s})$ \\
\hline
\end{tabular}

a) ${ }^{1} \mathrm{H}$ - and ${ }^{13} \mathrm{C}-\mathrm{NMR}$ assignments in $\mathrm{MeOH}-d_{4}$.

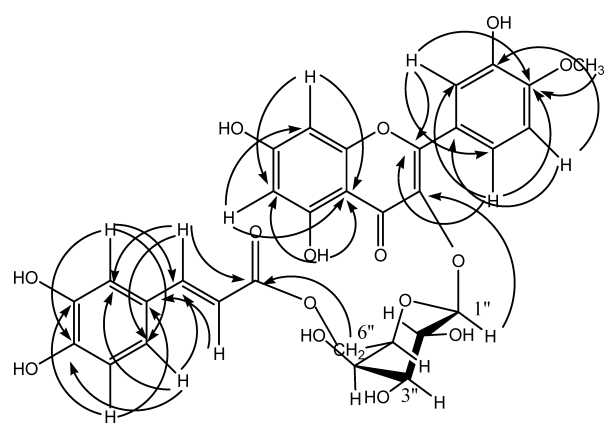

Fig. 2. Main HMBC Correlations of Compund $\mathbf{1}$

site of glycosidation was at the $\mathrm{C}-3$ position of tamarixetin. Compare with the isorhamnetin 3-O-(6" $O-E$-caffeoyl)- $\beta$-Dgalactopyranoside, ${ }^{10}$ the difference is the substituted B-ring, and it is flavonol $3^{\prime}$-methoxy-4'-hydroxy substituted B-ring. Therefore, the structure was determined as tamarixetin 3-O$\left(6^{\prime \prime}-O\right.$ - $E$-caffeoyl)- $\beta$-D-galactopyranoside.

Compound 2 was obtained as yellow powder. The molecular formula was established as $\mathrm{C}_{18} \mathrm{H}_{16} \mathrm{O}_{5}$ by HR-EI-MS. The IR spectrum showed characteristic absorption $\left(v_{\max }\right.$ $3500,2945,1600,1510,1465 \mathrm{~cm}^{-1}$ ) of an unsaturated homoisoflavone containing hydroxyl group. ${ }^{11)}$ The ${ }^{1} \mathrm{H}-\mathrm{NMR}$
Table 2. ${ }^{13} \mathrm{C}-\mathrm{NMR}\left(125 \mathrm{MHz}, \mathrm{DMSO}-d_{6}\right)$ Data of Compounds $\mathbf{2}-\mathbf{6}$

\begin{tabular}{|c|c|c|c|c|c|}
\hline No. & 2 & 5 & 3 & 4 & 6 \\
\hline 2 & 75.12 & 67.78 & 67.23 & 67.52 & 67.58 \\
\hline 3 & 127.13 & 128.63 & 127.36 & 128.79 & 129.13 \\
\hline 4 & 181.83 & 179.77 & 184.46 & 179.47 & 180.15 \\
\hline $4 a$ & 116.51 & 115.27 & 101.89 & 114.23 & 115.08 \\
\hline 5 & 122.96 & 123.06 & 164.28 & 129.37 & 118.35 \\
\hline 6 & 110.73 & 111.04 & 96.26 & 111.09 & 110.43 \\
\hline 7 & 156.78 & 156.94 & 166.83 & 164.57 & 152.34 \\
\hline 8 & 134.90 & 135.03 & 95.00 & 102.39 & 132.69 \\
\hline $8 a$ & 155.37 & 155.10 & 162.14 & 162.44 & 150.34 \\
\hline 9 & 139.11 & 135.46 & 136.41 & 135.19 & 135.17 \\
\hline $1^{\prime}$ & 126.83 & 126.45 & 126.36 & 126.48 & 126.54 \\
\hline $2^{\prime}, 6^{\prime}$ & 132.80 & 132.24 & 132.70 & 132.24 & 132.12 \\
\hline $3^{\prime}, 5^{\prime}$ & 113.33 & 114.29 & 114.57 & 114.28 & 114.29 \\
\hline $4^{\prime}$ & 160.43 & 160.31 & 160.77 & 160.24 & 160.23 \\
\hline $4^{\prime}-\mathrm{OCH}_{3}$ & 55.12 & 55.33 & 55.55 & 55.31 & 55.32 \\
\hline $8-\mathrm{OCH}_{3}$ & 60.13 & 60.28 & & & \\
\hline
\end{tabular}

Table 3. ${ }^{1} \mathrm{H}-\mathrm{NMR}\left(500 \mathrm{MHz}, \mathrm{DMSO}-d_{6}\right)$ Data of Compounds 2 - 6

\begin{tabular}{cccccc}
\hline \hline No. & $\mathbf{2}$ & $\mathbf{5}$ & $\mathbf{3}$ & $\mathbf{4}$ & $\mathbf{6}$ \\
\hline 2 & 5.05 & 5.41 & 5.35 & 5.35 & 5.35 \\
5 & 7.49 & 7.49 & - & 7.72 & 7.25 \\
6 & 6.59 & 6.61 & 5.88 & 6.52 & 6.55 \\
8 & - & - & 5.92 & 6.31 & - \\
9 & 7.02 & 7.64 & 7.70 & 7.62 & 7.63 \\
$2^{\prime}, 6^{\prime}$ & 7.89 & 7.41 & 7.43 & 7.39 & 7.41 \\
$3^{\prime}, 5^{\prime}$ & 6.93 & 7.04 & 7.06 & 7.03 & 7.05 \\
$4^{\prime}-\mathrm{OCH}_{3}$ & 3.78 & 3.81 & 3.82 & 3.81 & 3.81
\end{tabular}

spectrum of the compound was very informative regarding its structure. In the aromatic region two protons appearing at $\delta 7.49(1 \mathrm{H}, \mathrm{d}, J=9.0 \mathrm{~Hz}), \delta 6.59(1 \mathrm{H}, \mathrm{d}, J=9.0 \mathrm{~Hz})$, could be assigned to H-5, H-6 respectively. The signals for two protons doublets $(J=7.5 \mathrm{~Hz})$ at $\delta 7.89$ were suggested for $\mathrm{H}-2^{\prime}$ and $\mathrm{H}-6^{\prime}$ and $\delta 6.93$ for $\mathrm{H}-3^{\prime}$ and $\mathrm{H}-5^{\prime}$. The signals at $\delta 7.02$ $(1 \mathrm{H})$ and $\delta 5.05(2 \mathrm{H})$ were assigned to $\mathrm{H}-9$ and $\mathrm{H}-2$ respectively. These signals confirmed the compound $\mathbf{2}$ as a derivative of 3-benzylidene-4-chromanone. ${ }^{12)}$ The position of the vinylic proton was indicative of a cis $(Z)$ double bond at $\mathrm{C}_{3}-\mathrm{C}_{9}$ as the corresponding signal for $c i s(Z)$-isomer appears at $(\delta 6.97)$ and $(\delta 4.99) .{ }^{13)}$ And the signal for $\mathrm{H}-2$ at $\delta 5.05$ $(2 \mathrm{H})$ showed a cross-peak with the signal of H-9 at $\delta 7.02$ $(1 \mathrm{H})$ in the NOESY spectrum. The ${ }^{1} \mathrm{H}-\mathrm{NMR}$ spectrums also revealed the presence of a hydroxyl ( $\delta$ 10.34), two methoxyl groups $(\delta 3.78,3.70)$ in the molecule. The hydroxyl group has been placed at C-7 and the methoxyl group at C-8, C-4' on the basis of ${ }^{1} \mathrm{H}-\mathrm{NMR}$ spectrum along with mass spectrum which showed characteristic signals at $\mathrm{m} / \mathrm{z} 146$ and 166 due to retroDiels-Alder cleavage of the molecule. The compound 2 was thus characterized as cis (Z)-7-hydroxy-8-methoxy-3(4-methoxybenzyl) chroman-4-one which is the cis $(Z)$ isomer of 8-methoxybonducellin, the structure was further confirmed from its ${ }^{13} \mathrm{C}$-NMR (see Table 2) and HMBC experimental data. Thus the compound $\mathbf{2}$ was unambiguously established and named 8-methoxyisobonducellin.

Compounds 3-6 were obtained as yellow needles. Compounds 3-6 gave yellow-orange coloration with a $10 \%$ $\mathrm{H}_{2} \mathrm{SO}_{4}$ reagent on TLC, and showed positive color reactions. They had absorption bands assignable to hydroxyl, conjugated carbonyl groups and aromatic rings in the IR spectra. 
The 3-benzylidenechroman-4-one system was established from analyses of their ${ }^{1} \mathrm{H}-\mathrm{NMR}$ spectra and the $E$-geometry of the $\Delta^{3,9}$ was clearly discerned by the characteristic chemical shifts of the C-9 proton at $\delta 7.6$ and C-2 proton at $\delta$ 5.3 ppm. ${ }^{11,13)}$ They were characterized as the known compounds: eucomin (3), ${ }^{11)}$ bonducellin (4), ${ }^{13)} 8$-methoxybonducellin (5), ${ }^{12)}$ and intricatinol $(\mathbf{6}),{ }^{14)}$ respectively from their spectral (UV, IR, 1D and 2D NMR and MS) data and by comparison of the values with those reported earlier for the compounds.

\section{Experimental}

General Melting points were determined on a Fisher-Johns apparatus and were uncorrected. Optical rotations were measured on a Perkin-Elmer 241 polarimeter. IR spectra were recorded on a Perkin-Elmer 983G spectrometer. NMR spectra were measured in DMSO- $d_{6}$ on a Bruker AM-500 spectrometer, using TMS as internal standard. NMR experiments included the HMQC, HMBC. Coupling constants ( $J$ values) were given in $\mathrm{Hz}$. An Autospec-Ultima ETOF spectrometer was used to record the FAB-MS and HR-FAB-MS. silica gel $60 \mathrm{H}\left(400-500\right.$ mesh) and silica gel $\mathrm{GF}_{254}$ sheets $(0.20-0.25 \mathrm{~mm})$ (both from Qingdao Haiyang Chemical Group Co., Qingdao, Shandong Province, People's Republic of China) were used for column chromatography and TLC, respectively.

Plant Material The stems of Caesalpinia millettii Hook. et ARN. were collected in Jinghong, Yunnan Province (China) in October 2005, identified by associate Prof. Chao-Zhong PENG. And a voucher specimen (No. 00324971) is deposited at the Institute of Botany, the Chinese Academy of Sciences (CLDIS), Beijing, China.

Extraction and Isolation Dried stems of C. millettii Hook. et ARN. $(7 \mathrm{~kg})$ were extracted with acetone under reflux $(3 \mathrm{~h} \times 3)$, and the extract was evaporated in vacuo to afford a crude extract $(400 \mathrm{~g})$. The crude extract was fractionated by VLC with hexane- $\mathrm{CHCl}_{3}-\mathrm{MeOH}(1: 1: 0 ; 0: 1: 0 ; 0: 4: 1$; $0: 1: 1 ; 0: 0: 1)$ gradient system to give five fractions.

Fr. $2(15 \mathrm{~g})$ was rechromatographed on silica gel column with hexane-EtOAc $(9: 1, \mathrm{v} / \mathrm{v})$ to give 85 subfractions. Subfraction $59-69$ $(87 \mathrm{mg})$ was further subjected to sephedax LH-20 with $\mathrm{MeOH}$ to give 2 $(30 \mathrm{mg}), 5(25 \mathrm{mg})$. Fr. 3 (78 g) was rechromatographed on silica gel column with $\mathrm{CHCl}_{3}-\mathrm{MeOH}(10: 0 \rightarrow 1: 1, \mathrm{v} / \mathrm{v})$ to give five subfractions. Fr. 3-2 $(500 \mathrm{mg})$ was subjected to column chromatography with hexane- $\mathrm{CHCl}_{3}$ $(9: 1, \mathrm{v} / \mathrm{v})$ to give $3(46 \mathrm{mg})$. Similarly, Fr. $3-3(750 \mathrm{mg})$ was separated by CC with hexane-EtOAc $(9: 1, \mathrm{v} / \mathrm{v})$ to give $4(6 \mathrm{mg})$. Fr. 3-5 $(200 \mathrm{mg})$ was separated by Sephadex LH-20 $(\mathrm{MeOH})$, followed by column chromatography with hexane-EtOAc $(7: 3, \mathrm{v} / \mathrm{v})$ to give $6(37 \mathrm{mg})$. Fr. $5(2 \mathrm{~g})$ was separated by Sephadex LH-20 (MeOH) to give $1(30 \mathrm{mg})$.

Compound (1): Yellow powder $(\mathrm{MeOH})$. IR $(\mathrm{KBr}): 3250(\mathrm{OH}), 1645$, 1604, 1508, 1456, 1361, 1298, 1274, 1197, $1170 \mathrm{~cm}^{-1}$. ${ }^{1} \mathrm{H}-\mathrm{NMR}(500 \mathrm{MHz}$, DMSO- $\left.d_{6}\right) \delta: 12.61(5-\mathrm{OH}), 10.78(-\mathrm{OH}), 9.70(-\mathrm{OH}), 9.55(-\mathrm{OH}), 9.12$ $(-\mathrm{OH}), 7.65\left(1 \mathrm{H}, \mathrm{dd}, J=8.5,2.0 \mathrm{~Hz}, \mathrm{H}-6^{\prime}\right), 7.50\left(1 \mathrm{H}, \mathrm{d}, J=2.0 \mathrm{~Hz}, \mathrm{H}-2^{\prime}\right)$, $7.34\left(1 \mathrm{H}, \mathrm{d}, J=16 \mathrm{~Hz}, \mathrm{H}-7^{\prime \prime \prime}\right), 7.19\left(1 \mathrm{H}, \mathrm{d}, J=2.0 \mathrm{~Hz}, \mathrm{H}-2^{\prime \prime \prime}\right), 6.91(1 \mathrm{H}, \mathrm{dd}$, $\left.J=8.0,2 \mathrm{~Hz}, \mathrm{H}-6^{\prime \prime \prime}\right), 6.80\left(1 \mathrm{H}, \mathrm{d}, J=8.5 \mathrm{~Hz}, \mathrm{H}-5^{\prime}\right), 6.76(1 \mathrm{H}, \mathrm{d}, J=8.0 \mathrm{~Hz}, \mathrm{H}-$ $\left.5^{\prime \prime \prime}\right), 6.34(1 \mathrm{H}, \mathrm{d}, J=2.0 \mathrm{~Hz}, \mathrm{H}-8), 6.23\left(1 \mathrm{H}, \mathrm{d}, J=16 \mathrm{~Hz}, \mathrm{H}-8^{\prime \prime \prime}\right), 6.10(1 \mathrm{H}, \mathrm{d}$, $J=2.0 \mathrm{~Hz}, \mathrm{H}-6), 5.39\left(1 \mathrm{H}, \mathrm{d}, J=8.0 \mathrm{~Hz}, \mathrm{H}-1^{\prime \prime}\right), 5.18(-\mathrm{OH}), 4.93(-\mathrm{OH})$, $4.71(-\mathrm{OH}), 4.08\left(1 \mathrm{H}, \mathrm{m}, \mathrm{H}-6^{\prime \prime}\right), 3.80\left(\mathrm{OCH}_{3}\right), 3.68\left(1 \mathrm{H}, \mathrm{m}, \mathrm{H}-5^{\prime \prime}\right), 3.66(1 \mathrm{H}$, $\left.\mathrm{m}, \mathrm{H}-4^{\prime \prime}\right), 3.57\left(1 \mathrm{H}, \mathrm{m}, \mathrm{H}-2^{\prime \prime}\right), 3.43\left(1 \mathrm{H}, \mathrm{m}, \mathrm{H}-3^{\prime \prime}\right) .{ }^{1} \mathrm{H}-(500 \mathrm{MHz})$ and ${ }^{13} \mathrm{C}-$ NMR (125 MHz) in DMSO- $d_{6}$ see Table 1. HR-FAB-MS (positive-ion) $m / z$ : 663.1311) $[\mathrm{M}+\mathrm{Na}]^{+}$(Calcd for $\mathrm{C}_{31} \mathrm{H}_{28} \mathrm{O}_{15} \mathrm{Na}$ : 663.1325).

Compound (2): Yellow powder. IR (KBr): $3500(\mathrm{OH}), 2945,1666(\mathrm{C}=\mathrm{O})$, 1600 (aromatic), 1510, 1465, 1440, 1396, 1284, 1259, 1178, 1083, 1207 , $835,786 \mathrm{~cm}^{-1} .{ }^{1} \mathrm{H}-\mathrm{NMR}\left(500 \mathrm{MHz}, \mathrm{DMSO}-d_{6}\right) \delta: 10.34(1 \mathrm{H}, \mathrm{s}, 7-\mathrm{OH})$, $7.89\left(2 \mathrm{H}, \mathrm{d}, J=7.5 \mathrm{~Hz}, \mathrm{H}-2^{\prime}, 6^{\prime}\right), 7.49(1 \mathrm{H}, \mathrm{d}, J=9 \mathrm{~Hz}, 5-\mathrm{H}), 7.02(1 \mathrm{H}, \mathrm{s}, 9-$ H), $6.93\left(2 \mathrm{H}, \mathrm{d}, J=7.5 \mathrm{~Hz}, \mathrm{H}-3^{\prime}, 5^{\prime}\right), 6.59(1 \mathrm{H}, \mathrm{d}, J=7.5 \mathrm{~Hz}, 6-\mathrm{H}), 5.05(2 \mathrm{H}$, s, 2-H), $3.78\left(3 \mathrm{H}, \mathrm{s}, \mathrm{OCH}_{3}\right), 3.70\left(3 \mathrm{H}, \mathrm{s}, \mathrm{OCH}_{3}\right) \cdot{ }^{13} \mathrm{C}-\mathrm{NMR}(125 \mathrm{MHz})$ in DMSO- $d_{6}$ see Table 2. HR-EI-MS $m / z: 312.0998[\mathrm{M}]^{+}\left(\right.$Calcd for $\mathrm{C}_{18} \mathrm{H}_{16} \mathrm{O}_{5}$ : 312.0997).

Compound (3): Yellow needles $\left(\mathrm{CHCl}_{3} / \mathrm{MeOH}\right) ; \mathrm{mp} 199-201{ }^{\circ} \mathrm{C} .{ }^{1} \mathrm{H}-$ NMR $\left(500 \mathrm{MHz}\right.$, DMSO- $\left.d_{6}\right) \delta: 7.70(1 \mathrm{H}, \mathrm{s}, 9-\mathrm{H}), 7.43\left(2 \mathrm{H}, \mathrm{d}, J=8.8 \mathrm{~Hz}, 2^{\prime}\right.$, $\left.6^{\prime}-\mathrm{H}\right), 7.06\left(2 \mathrm{H}, \mathrm{d}, J=8.8 \mathrm{~Hz}, 3^{\prime}, 5^{\prime}-\mathrm{H}\right), 5.92(1 \mathrm{H}, \mathrm{d}, J=2 \mathrm{~Hz}, 8-\mathrm{H}), 5.88$ $(1 \mathrm{H}, \mathrm{d}, J=2 \mathrm{~Hz}, 6-\mathrm{H}), 5.35(2 \mathrm{H}, \mathrm{s}, 2-\mathrm{H}), 3.82\left(3 \mathrm{H}, \mathrm{OCH}_{3}\right) \cdot{ }^{13} \mathrm{C}-\mathrm{NMR}$ $\left(125 \mathrm{MHz}\right.$, DMSO- $d_{6}$ ) see Table 2. EI-MS $m / z: 298[\mathrm{M}]^{+}$

Compound (4): Yellow needles $\left(\mathrm{CHCl}_{3} / \mathrm{MeOH}\right)$; mp $207^{\circ} \mathrm{C} .{ }^{1} \mathrm{H}-\mathrm{NMR}$ $\left(500 \mathrm{MHz}, \mathrm{DMSO}-d_{6}\right) \delta: 10.61(7-\mathrm{OH}), 7.72(1 \mathrm{H}, \mathrm{d}, J=8.0 \mathrm{~Hz}, 5-\mathrm{H}), 7.62$ $(1 \mathrm{H}, \mathrm{s}, 9-\mathrm{H}), 7.39\left(2 \mathrm{H}, \mathrm{d}, J=8.5 \mathrm{~Hz}, 2^{\prime}, 6^{\prime}-\mathrm{H}\right), 7.03\left(2 \mathrm{H}, \mathrm{d}, J=8.5 \mathrm{~Hz}, 3^{\prime}, 5^{\prime}-\right.$ $\mathrm{H}), 6.52(1 \mathrm{H}, \mathrm{dd}, J=8.5,2 \mathrm{~Hz}, 6-\mathrm{H}), 6.31(1 \mathrm{H}, \mathrm{d}, J=2.0 \mathrm{~Hz}, 8-\mathrm{H}), 5.35(2 \mathrm{H}$, d, $J=1.5 \mathrm{~Hz}, 2-\mathrm{H}), 3.81\left(3 \mathrm{H}, \mathrm{s}, \mathrm{OCH}_{3}\right) \cdot{ }^{13} \mathrm{C}-\mathrm{NMR}\left(125 \mathrm{MHz}, \mathrm{DMSO}-d_{6}\right)$ see Table 2. EI-MS $m / z: 282[\mathrm{M}]^{+}$.

Compound (5): Yellow needles $\left(\mathrm{CHCl}_{3} / \mathrm{MeOH}\right) ; \mathrm{mp} 124-125^{\circ} \mathrm{C} .{ }^{1} \mathrm{H}-$ NMR $\left(500 \mathrm{MHz}\right.$, DMSO- $\left.d_{6}\right) \delta: 10.36(-\mathrm{OH}), 7.64(1 \mathrm{H}, \mathrm{s}, 9-\mathrm{H}), 7.49(1 \mathrm{H}, \mathrm{d}$, $J=9.5 \mathrm{~Hz}, 5-\mathrm{H}), 7.41\left(2 \mathrm{H}, \mathrm{d}, J=8.5 \mathrm{~Hz}, 2^{\prime}, 6^{\prime}-\mathrm{H}\right), 7.04(2 \mathrm{H}, \mathrm{d}, J=8.5 \mathrm{~Hz}$, $\left.3^{\prime}, 5^{\prime}-\mathrm{H}\right), 6.61(1 \mathrm{H}, \mathrm{d}, J=9.5 \mathrm{~Hz}, 6-\mathrm{H}), 5.41(2 \mathrm{H}, \mathrm{s}, 2-\mathrm{H}), 3.81\left(3 \mathrm{H}, \mathrm{OCH}_{3}\right)$, $3.70\left(3 \mathrm{H}, \mathrm{OCH}_{3}\right) \cdot{ }^{13} \mathrm{C}-\mathrm{NMR}\left(125 \mathrm{MHz}, \mathrm{DMSO}-d_{6}\right)$ see Table 2 . EI-MS $m / z$ : $312[\mathrm{M}]^{+}$.

Compound (6): Yellow needles $\left(\mathrm{CHCl}_{3} / \mathrm{MeOH}\right) ; \mathrm{mp} 196-197^{\circ} \mathrm{C} .{ }^{1} \mathrm{H}-$ NMR $\left(500 \mathrm{MHz}\right.$, DMSO- $\left.d_{6}\right) \delta: 7.63(1 \mathrm{H}, \mathrm{s}, 9-\mathrm{H}), 7.41\left(2 \mathrm{H}, \mathrm{d}, J=9 \mathrm{~Hz}, 2^{\prime}\right.$, $\left.6^{\prime}-\mathrm{H}\right), 7.25(1 \mathrm{H}, \mathrm{d}, J=9 \mathrm{~Hz}, 5-\mathrm{H}), 7.05\left(2 \mathrm{H}, \mathrm{d}, J=9 \mathrm{~Hz}, 3^{\prime}, 5^{\prime}-\mathrm{H}\right), 6.55(1 \mathrm{H}$, d, $J=9 \mathrm{~Hz}, 6-\mathrm{H}), 5.35(2 \mathrm{H}, \mathrm{d}, J=1.5 \mathrm{~Hz}, 2-\mathrm{H}), 3.81\left(3 \mathrm{H}, \mathrm{s}, \mathrm{OCH}_{3}\right) .{ }^{13} \mathrm{C}-$ NMR (125 MHz, DMSO- $d_{6}$ ) see Table 2. EI-MS m/z: $298[\mathrm{M}]^{+}$.

Acknowledgements The authors are grateful to the staff of the Analytical Center of the Institute of Materia Medica, Chinese Academy of Medical Sciences \& Peking Union Medical College for the measurement of NMR and MS.

\section{References}

1) Flora of China Editorial Committee, P. R. C. (ed.), "Flora of China," Vol. 39, Science Press, Beijing, 1988, p. 100.

2) Kitagawa I., Simanjuntak P., Watano T., Shibuya H., Fujii S., Yamagata Y., Kobayashi M., Chem. Pharm. Bull., 42, 1798-1802 (1994).

3) Patil A. D., Freyer A. J., Webb R. L., Zuber G., Reichwein R., Bean M. F., Faucette L., Johnson R. K., Tetrahedron, 53, 1583-1592 (1997).

4) Jiang R. W., Ma S. C., But P. P., Mak T. C. W., J. Nat. Prod., 64, $1266-1272(2001)$.

5) Jiang R. W., Ma S. C., He Z. D., Huang X. S., But P. P. H., Wang H., Chan S. P., Ooi V. E. C., Xu H. X., Mak T. C. W., Bioorg. Med. Chem., 10, 2161-2170 (2002).

6) Ragasa C. Y., Hofilena J. G, Rideout J. A., J. Nat. Prod., 65, 11071110 (2002).

7) Linn T. Z., Awale S., Tezuka Y., Banskota A. H., Kalauni S. K., Attamimi F., Ueda J. Y., Asih P. B. S., Syafruddin D., Tanaka K., Kadota S., J. Nat. Prod., 68, 706-710 (2005).

8) Jiang R. W., But P. P., Ma S. C., Ye W. C., Chan S. P., Mak T. C. W., Tetrahedron Lett., 43, 415- 418 (2002).

9) Harborne J. B., "1 H Nuclear Magnetic Resonance Spectroscopy of Flavonoids and Their Glycosides in Hexadeuterodimethylsulfoxide," Chapman \& Hall, CRC Press, London, 1994, pp. 441- 497.

10) Calzada F., Roberto C. R., Mata R., J. Nat. Prod., 64, 671-673 (2001).

11) Böhler P., Tamm C., Tetrahedron Lett., 36, 3479-3483 (1967).

12) Mcpherson D. D., Cordell G. A., Soejarto D. D., Pizzuto J. M., Fong H. H. S., Phytochemistry, 22, 2835-2838 (1983).

13) Srinivas K. V. N. S., Rao K. Y., Mahender I., Das B., Krishna K. V. S. R., Kishore K. H., Murty U. S. N., Phytochemistry, 63, 789-793 (2003).

14) Wall M. E., Wani M. C., Manikumar G., Talor H., Mcgivney N., J. Nat. Prod., 52, 774-778 (1989). 